\title{
Canadian Consensus Guidelines on Use of Amyloid Imaging in Canada: Update and Future Directions from the Specialized Task Force on Amyloid imaging in Canada
}

\author{
Robert Laforce Jr., Pedro Rosa-Neto, Jean-Paul Soucy, Gil D. Rabinovici, \\ Bruno Dubois, S. Gauthier, on behalf of the consensus meeting participants
}

\begin{abstract}
Positron emission tomography (PET) imaging of brain amyloid beta is now clinically available in several countries including the United States and the United Kingdom, but not Canada. It has become an established technique in the field of neuroimaging of aging and dementia, with data incorporated in the new consensus guidelines for the diagnosis of Alzheimer disease and predementia Alzheimer's disease-related conditions. At this point, there are three US Food and Drug Administration- and European Union-approved tracers. Guided by appropriate use criteria developed in 2013 by the Alzheimer's Association and the Society of Nuclear Medicine and Molecular Imaging, the utility of amyloid imaging in medical practice is now supported by a growing body of research. In this paper, we aimed to provide an update on the 2012 Canadian consensus guidelines to dementia care practitioners on proper use of amyloid imaging. We also wished to generate momentum for the industry to submit a new drug proposal to Health Canada. A group of local, national, and international dementia experts and imaging specialists met to discuss scenarios in which amyloid PET could be used appropriately. Peer-reviewed and published literature between January 2004 and May 2015 was searched. Technical and regulatory considerations pertaining to Canada were considered. The results of a survey of current practices in Canadian dementia centers were considered. A set of specific clinical and research guidelines was agreed on that defines the types of patients and clinical circumstances in which amyloid PET could be used in Canada. Future research directions were also outlined, notably the importance of studies that would assess the pharmaco-economics of amyloid imaging.
\end{abstract}

RÉSUMÉ: Lignes directrices consensuelles quant à l'utilisation de l'imagerie amylö̈de au Canada : mise à jour et pistes pour l'avenir proposées par le Groupe d'étude canadien sur l'imagerie amylö̈de. La tomographie par émission de positons (TEP), examen d'imagerie de la protéine bêta-amylö̈de du cerveau, est désormais disponible sur le plan clinique dans de nombreux pays, dont les États-Unis et le Royaume-Uni, mais pas encore au Canada. La TEP s'est imposée comme une technique reconnue dans le champ de la neuro-imagerie associé au vieillissement et aux démences. Les données ainsi recueillies ont été intégrées à de nouvelles lignes directrices consensuelles en ce qui concerne le diagnostic de la maladie d'Alzheimer et des problèmes liés à sa phase pré-démentielle. À l'heure actuelle, trois traceurs ont été approuvés par la Food and Drug Administration des États-Unis ainsi que par l'Union européenne. S'appuyant sur des critères d'utilisation appropriés (« AUC » en anglais) élaborés en 2013 par l'Alzheimer's Association et la Society of Nuclear Medicine and Molecular Imaging, l'utilité de l'imagerie amylö̈de en médecine est dorénavant étayée par un nombre grandissant de travaux de recherche. Dans cet article, nous entendons proposer une mise à jour des lignes directrices consensuelles établies au Canada en 2012 quant à une utilisation appropriée de l'imagerie amyloïde. Ces lignes directrices sont destinées aux professionnels de la santé appelés à traiter la démence. Nous souhaitons également susciter un élan dans l'industrie pharmaceutique visant à soumettre des propositions de nouveaux médicaments à Santé Canada. À cet égard, un groupe d'experts nationaux et internationaux en matière de démence, de même que des spécialistes en imagerie, se sont rencontrés afin d'examiner les situations en vertu desquelles la TEP pourrait être utilisée de manière appropriée. Pour notre part, nous avons d'abord effectué une revue de la littérature scientifique soumise à examen collégial et publiée entre janvier 2004 et mai 2015 . Nous nous sommes penchés ensuite sur les considérations techniques et réglementaires propres au Canada ainsi que sur les résultats d'un sondage portant sur les pratiques en vigueur dans les centres canadiens de soins de la démence. Nous avons ainsi convenu d'un ensemble de lignes directrices spécifiques concernant le travail clinique et la recherche. Ces lignes directrices visent à définir les types de patients et les circonstances cliniques en vertu desquels la TEP pourrait être utilisée au Canada. Enfin, de futurs axes de recherche ont également été soulignés, en particulier l'importance d'études pouvant évaluer la dimension pharmaco-économique de l'imagerie amyloïde.

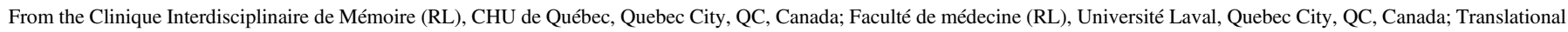

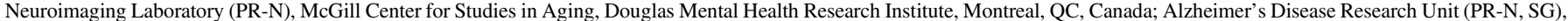

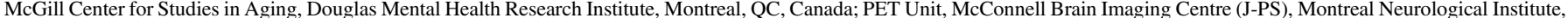
McGill University, Montreal, QC, Canada; PERFORM Centre (J-PS), Concordia University, Montreal, QC, Canada; Médecine Nucléaire (J-PS), Centre hospitalier de l'université de

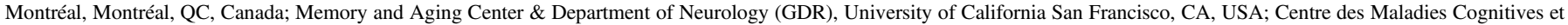
Comportementales (BD), Institut du Cerveau et de la Moelle épinière, Paris, France; Université Pierre et Marie Curie-Paris 6 (BD), AP-HP, Hôpital de la Salpêtrière, Paris, France. Received August 31, 2015. Final Revisions SubmitTed November 25, 2015.

Correspondence to: Dr. Robert Laforce Jr., Neurologue et Neuropsychologue, Professeur Agrégé, Faculté de Médecine, Université Laval, Clinique Interdisciplinaire de Mémoire, Département des Sciences Neurologiques, CHU de Québec, 1401, 18ième rue, Québec, Canada, G1J 1Z4. Email: robert.laforce@ fmed.ulaval.ca 
Keywords: Amyloid imaging, Alzheimer's Disease, Atypical Dementia, Biomarkers, Consensus Guidelines, MCI, PET

Since 1989, four Canadian Consensus Conferences on the Diagnosis and Treatment of Dementia (CCCDTD) have been held. ${ }^{1-4}$ Of those, the 4 th CCCDTD $^{3}$ focused on updating previous diagnostic approaches to Alzheimer's disease (AD) taking into account revised diagnostic criteria proposed by the International Working Group ${ }^{5,6}$ and the recommendations made by the National Institute on Aging - Alzheimer Association (AA) workgroups. ${ }^{7-9}$ The focus of CCCDTD4 and of two accompanying papers ${ }^{10,11}$ was largely on neuroimaging and other biomarkers; nine recommendations on amyloid positron emission tomography (PET) were made, most of which are now outdated. In this paper, we aimed to provide updated Canadian guidelines to dementia care practitioners on proper use of amyloid imaging as formulated by the Specialized Task force on Amyloid imaging in Canada (STAC). We also wished to generate momentum for the industry to submit a new drug proposal to Health Canada so that this revolutionary technique could become part of our clinical armamentarium under precise and well-defined indications.

\section{Amyloid Imaging: From 2004 to Today}

Since Klunk's publication on Pittsburgh compound B $(\mathrm{PiB})$ in 2004, ${ }^{12}$ PET using amyloid ligands has revolutionized AD research, leading to improved models of disease pathogenesis, providing evidence for a long preclinical disease phase, and stimulating therapeutic trials aimed at delaying or preventing the symptomatic phase of AD. ${ }^{13,14}$ For example, amyloid imaging has served as a secondary outcome measure in $\mathrm{AD}$ clinical trials with diseasemodifying agents such as the antiamyloid monoclonal antibodies bapineuzumab and solanezumab. ${ }^{15,16}$ Brain amyloid reduction and slowing of cognitive decline were found after 1 year of treatment with aducanumab, a human immunoglobulin G1 monoclonal antibody against a conformational epitope found on amyloid beta (A $\beta) .{ }^{17}$ Finally, its added value also lies in subject selection for clinical trials given an approximate $15 \%$ amyloid negative rate.

Beyond research, amyloid imaging has demonstrated great potential as a diagnostic tool largely because it allows in vivo detection of amyloid plaques, a core pathologic feature of $\mathrm{AD} .^{12} \mathrm{It}$ is now an established technique in the field of neuroimaging of aging and dementia, with data incorporated in the most recent consensus guidelines for the diagnosis of $\mathrm{AD}^{7}$ and predementia $\mathrm{AD}$-related conditions. ${ }^{8,9}$ There currently are three US Food and Drug Administration and European Union-approved, fluorine-18-labeled, tracers available for clinical use: florbetapir since $2012,{ }^{18}$ flutemetamol since $2013,{ }^{19}$ and florbetaben since $2014 .{ }^{20}$ Converging evidence on the diagnostic utility of that technique has rapidly accumulated. ${ }^{21-29}$ Still, no tracer has been approved for clinical use in Canada so far.

\section{Amyloid PET in Cognitively Normal Elderly Individuals and Various Clinical Populations}

\section{Cognitively Normal Elderly Individuals}

Cognitively normal elderly individuals (CNs) show elevated $\mathrm{PiB}$ binding in $10 \%$ to $34 \%$ of cases, a proportion similar to observed rates of amyloid pathology in autopsy studies. ${ }^{30}$ Increasing age and the presence of the apolipoprotein E $\varepsilon 4$ allele are the major predictors of $\mathrm{PiB}$ positivity in $\mathrm{CN} .{ }^{31,32}$ Recent findings in persons without dementia or mild cognitive impairment ${ }^{8,33,34}$ suggest that amyloid deposition is associated with very subtle cognitive deficits, especially among apolipoprotein E $\varepsilon 4$ carriers. ${ }^{35}$ Interestingly, recent data suggest that the cooccurrence of $A \beta$ and neurodegeneration (hippocampus volume and glucose metabolism) is associated with cognitive decline in CNs. ${ }^{36}$

The significance of a positive amyloid scan in CNs still remains uncertain, but cross-sectional studies have shown "AD-like" brain changes (hippocampal and temporo-parietal atrophy), ${ }^{37}$ whereas early longitudinal data have strengthened the notion that many (although probably not all) are in a "preclinical" phase of AD." Recently, a group of researchers compared the ability of molecular biomarkers for $\mathrm{AD}$, including amyloid imaging and cerebrospinal fluid (CSF) biomarkers $\left(\mathrm{A} \beta_{1-42}\right.$, tau, ptau181, tau/A $\beta_{1-42}$, ptau181/A $\left.\beta_{1-42}\right)$, to predict time to incident cognitive impairment among cognitively normal adults aged 45 to 88 years and followed for up to 7.5 years. Results indicated that all AD biomarkers studied predicted incident cognitive impairment and supported the hypothesis that biomarkers signal underlying AD pathology at least several years before the appearance of dementia symptoms. ${ }^{38}$

From a diagnostic perspective, the significant number of amyloid-positive CNs emphasizes that amyloid positivity is not synonymous with $\mathrm{AD}$, and that amyloid scans cannot replace a detailed clinical evaluation. At present, there is no clinical indication for amyloid imaging in CNs, though this will remain an area of active research in coming years, particularly with the advent of amyloid-lowering therapies, which might be most effective if initiated in the presymptomatic disease stage. ${ }^{39,40}$

\section{Mild Cognitive Impairment}

Current data in mild cognitive impairment (MCI) $8,33,34$ indicates that amyloid imaging provides prognostic information, presumably by identifying patients with underlying AD pathology. ${ }^{41}$ As a group, $52 \%$ to $87 \%$ of MCI subjects show elevated $\mathrm{PiB}$ binding in a regional distribution similar to that observed with $\mathrm{AD} .{ }^{30}$ In longitudinal studies, 1-year conversion rates to $\mathrm{AD}$ range from $33 \%$ to $47 \%$ in PiB-positive MCI subjects, whereas virtually no conversions are seen in PiB-negative subjects. ${ }^{42}$ In a longitudinal study, ${ }^{43}$ the authors compared baseline amyloid deposition between MCI converters and nonconverters in 31 subjects followed over 3 years. The conversion rate was $82 \%$ in those with increased $\mathrm{PiB}$ uptake, but only $7 \%$ in PiB-negative subjects. Results from the Australian Imaging Biomarkers and Lifestyle study of aging on 87 participants with MCI (age, $73.7 \pm 8.27$ ) showed that $59 \%$ had progressed to probable AD over 3 years. ${ }^{44}$ Multivariate analysis showed $\beta$-amyloid imaging as the variable most strongly associated with progression. Almost all amnestic MCI subjects with a positive amyloid scan developed AD. Altogether, the literature clearly suggests that PiB-positive amnestic MCI patients are likely to have early $\mathrm{AD}$, and amyloid imaging will 
help in risk stratification and selection of patients who may benefit from experimental disease-specific therapies. Similar to normal aging, positivity of both $\mathrm{A} \beta$ and neurodegeneration biomarkers in MCI can further stratify risk of imminent conversion to dementia.

\section{Alzheimer's Disease}

Most studies in $\mathrm{AD}^{6,7}$ have found very high (90\% or greater) PiB-PET positivity, with a pattern that closely mirrors the distribution of plaques found at autopsy. ${ }^{31}$ Tracer binding is diffuse and symmetric, with high uptake consistently found in prefrontal cortex, precuneus and posterior cingulate cortex, followed closely by lateral parietal and lateral temporal cortex, and striatum. Studies in atypical clinical presentations of AD have shown that amyloid deposition is more common in the logopenic variant of primary progressive aphasia than in nonfluent or semantic variants, ${ }^{45}$ supporting the hypothesis that logopenic variant of primary progressive aphasia is often associated with underlying AD. Others have detected high $\mathrm{PiB}$ binding in patients with posterior cortical atrophy, a visuospatial/biparietal clinical syndrome often caused by AD. ${ }^{46}$ Much like ${ }^{18}$ F-fluorodeoxyglucose-PET (FDG-PET), ${ }^{47}$ amyloid imaging will probably not add value to the diagnostic workup of patients with straightforward clinical AD, but is likely to be useful in patients with atypical complex presentations or early age-of-onset dementia (see the following two sections).

\section{The Frontotemporal Lobar Degeneration Spectrum of Disorders}

Considering that the frontotemporal lobar degeneration spectrum of disorders (FTLD) and AD are the leading causes of early age-of-onset dementia, ${ }^{48}$ that distinguishing the two during life can be clinically challenging but is also important, ${ }^{49}$ and that $\mathrm{A} \beta$ plaques are not part of the FTLD pathologic spectrum, several authors have argued for a valuable role of amyloid imaging in the differential diagnosis of these conditions. Small case series have reported low rates of $\mathrm{PiB}$ positivity $(0 \%$ to $15 \%)$ and florbetaben positivity $(9 \%)$ in FTLD. ${ }^{50}$ Recently, results from the largest study currently available on the diagnostic utility of amyloid PET in FTLD showed, in $62 \mathrm{AD}$ and 45 FTLD patients, that PiB visual reads had a higher sensitivity for AD than FDG-PET, with similar specificity. ${ }^{23} \mathrm{PiB}$ outperformed FDG in classifying patients with known histopathology, and visual reads showed higher inter-rater reliability and agreement than for FDG, suggesting it was the more accurate and precise technique.

\section{Complex, Atypical Patients With an Uncertain Diagnosis}

The clinical diagnosis of AD has only moderate sensitivity and specificity when compared with the pathological cause of dementia as determined at autopsy. ${ }^{51}$ Misdiagnosis rates are even higher in complex, atypical patients with an uncertain diagnosis, approaching $30 \%{ }^{26} \mathrm{~A}$ growing body of literature supports the clinical utility of amyloid imaging for the differential diagnosis of atypical patients with an uncertain diagnosis. ${ }^{21,22,24-29,52}$ This has major implications for a cohort of individuals who are often younger than 65 years of age and still active in the workforce. Indeed, several dementia experts have argued that an accurate diagnosis helps direct therapy (i.e. avoid unnecessary or undesired cholinesterase inhibitors or memantine prescriptions), determine a better care plan (which considers patient safety and minimizes the risk of preventable complications), and enable patients to participate in legal and financial planning.

Recently, a group of Canadians researchers investigated the clinical utility of amyloid PET in the differential diagnosis of atypical cases and its impact on caregivers in the context of a tertiary memory clinic. ${ }^{29}$ Using the amyloid tracer ${ }^{18}$ F-NAV4694, ${ }^{53-55}$ they prospectively scanned 25 patients (mean age, 59.3 years; standard deviation [SD], 5.8; and mean Mini-Mental State Examination, 21.6; SD 6.2) with an atypical syndrome as determined by dementia experts. All patients had a full workup (i.e. history, examination, blood tests, neuropsychology, magnetic resonance imaging [MRI], and FDG-PET), yet no certain diagnosis could be arrived at following that investigation. Amyloid PET was either positive or negative based on qualitative and quantitative reads by two qualified independent expert nuclear medicine specialists. Physicians rated whether amyloid PET was associated with a change in diagnosis and altered management. They also reported their degree of confidence in diagnosis before and after amyloid PET. Caregivers were met 3 months after having been told of the diagnosis and completed a 21-item Likert scale questionnaire along with a 1-hour interview designed to assess the impact of the amyloid scan. The cohort was $48 \%$ amyloid positive and $52 \%$ amyloid negative. Inter-rater reliability was $100 \%$. Amyloid PET was associated with a diagnostic change in $36 \%$ (9/25) of cases (24\% changed from AD to non-AD and $12 \%$ from non-AD to AD). There was a significant increase $(40 \%)$ in diagnostic confidence following the scan. Altogether, this study corroborated recent findings and suggested an additive role for amyloid PET in atypical cases with an unclear diagnosis despite the detailed workup of a tertiary memory clinic. Amyloid PET increased diagnostic confidence and generated significant alterations in management in almost three-quarters of cases. Furthermore, the overall process was very well received by caregivers, reducing anxiety and depressive symptomatology as well as increasing quality time spent with their loved ones.

\section{Other Clinical Conditions}

Other clinical conditions studied with amyloid PET include vascular cognitive impairment, cerebral amyloid angiopathy, Parkinson's disease dementia, and dementia with Lewy bodies (DLB). In one study on vascular cognitive impairment, authors found that $69 \%$ of patients were PiB-negative. ${ }^{56} \mathrm{High} \mathrm{PiB}$ binding rates were found in nondemented patients with cerebral amyloid angiopathy. ${ }^{57}$ Most studies showed higher amyloid plaques in DLB than in Parkinson's disease dementia or nondemented PD patients and, in some, $\mathrm{PiB}$ positivity was associated with more rapid disease progression. ${ }^{58}$ The high frequency of plaques and high rates of positive scans in DLB suggest that amyloid PET is unlikely to be helpful in differentiating DLB from AD.

\section{Amyloid Pet in Clinical Practice Today: Are We READY YET?}

The translation from the research setting into the clinic has progressed steadily. ${ }^{59-61}$ For example, a group recently determined the sensitivity and specificity of amyloid PET with flutemetamol using neuropathologically determined neuritic plaque levels and showed high sensitivity and specificity in an end-of-life population. ${ }^{18,62,63}$ In an effort to guide clinicians, the Society of Nuclear Medicine and Molecular Imaging (SNMMI) and the AA jointly published criteria for the appropriate use of amyloid 
PET. ${ }^{59,61}$ Over the past 3 years, almost 10 reports on the practical clinical applications of amyloid imaging have been published, and their conclusions indicated a significant role in orienting treatment (i.e. deciding whether to initiate or discontinue AD symptomatic medications) and a positive impact on caregivers. ${ }^{21,22,24-29,52}$ Finally, much is expected from the soon-to-be launched Imaging Dementia - Evidence for Amyloid Scanning (IDEAS) Study, a $\$ 100$ million open-label longitudinal cohort effort on approximately 18,500 US Medicare beneficiaries. In this venture, diagnostically uncertain cases of MCI and atypical dementia will be scanned to determine whether knowledge of amyloid status leads to significant changes in patient management and if this translates into improved medical outcomes.

In light of the outstanding worldwide momentum surrounding the utility of amyloid imaging in clinical practice, and the fact that Canada lags behind on approval of the technique by health authorities, we first aimed to provide updated Canadian guidelines to dementia care practitioners on proper use of amyloid imaging. Second, we wished to generate momentum for the industry to submit a new drug proposal to Health Canada.

\section{Methods}

The STAC met in Montreal, QC, Canada on May 14, 2015, to update the 2012 Canadian consensus guidelines, ${ }^{3}$ which incorporated nine recommendations on amyloid PET, most of which are now outdated. The meeting included all members of the STAC (a group of local, national, and international dementia experts and imaging specialists; see Appendix A) as well as consensus meeting participants (clinical and academic; see Appendix A), and leading representatives from the molecular imaging and imaging software industries. Technical and regulatory considerations pertaining to Canada were discussed with a member of Health Canada who attended the meeting. Peer-reviewed and published literature between January 2004 and May 2015 was searched before the meeting. A survey of current diagnostic practices in Canadian dementia centers was also presented to allow focused discussions on Canadian medical practices. In brief, we discussed indications and, just as importantly nonindications, of amyloid imaging based on clinical and nonclinical scenarios with variables including symptoms (typical and atypical), clinical settings, clinical contexts, evidence of cognitive deficits, family history, knowledge of AD genetic risk, and age.

Although previous CCCDTDs had used the evidence grading system developed by the Canadian Task Force on Preventive Health Care, for this iteration we attempted to follow, where possible, the Grading of Recommendations Assessment, Development and Evaluation system in keeping with current recommendations for the conduct of consensus conferences. ${ }^{64}$ Each participant was allowed to take part in the discussion. Consensus was defined as $80 \%$ or more conference participants being in agreement with a recommendation. Partial consensus was defined as $60 \%$ to $79 \%$ being in agreement. Recommendations reaching consensus are listed in Tables 1 and 2. Recommendations reaching only partial or no consensus are only mentioned in the Discussion section.

\section{RESULTS}

A set of updated guidelines were agreed on that define the types of patients and clinical circumstances in which amyloid PET could be used in Canada (Table 1). Future research directions were also outlined, notably the importance of studies that would assess the pharmaco-economics of such diagnostic procedure (Table 2).

\section{Survey of the Availability and Use of Biomarkers in Canada}

Three months before the meeting, a survey was sent to all Canadian dementia centers. A total of 27 respondents (British Columbia, 3; Alberta, 3; Saskatchewan, 1; Ontario, 7; Quebec, 10; Maritimes, 3) provided answers mainly to two questions: (1) How many early-onset atypical dementia cases do you see per month, and (2) Which advanced diagnostic techniques do you use in practice beyond clinical history, physical examination, standard laboratory tests, and basic computed tomography imaging (i.e. MRI, hippocampal volumetry, molecular imaging, CSF A $\beta_{1-42}$ and tau). Results indicated that an average of 5,8 (SD: 5,4) earlyonset atypical dementia cases per clinician were seen per month. More than $80 \%$ use MRI. Only clinicians from Quebec use FDGPET in clinical practice, whereas single-photon emission computed tomography is used by a majority of clinicians outside Quebec, because of regionally specific provincial reimbursement issues. Only $20 \%$ of clinicians use CSF measures. Less than $11 \%$ prescribe acetylcholine-esterase inhibitors to atypical cases with uncertain diagnoses. Finally, in $85 \%$ of cases, clinicians reported that knowledge of the amyloid status of their atypical patient would change their therapeutic approach. Some clinicians also reported sending patients to the United States for an amyloid scan.

\section{DISCUSSION}

Amyloid PET is now an established neuroimaging technique with data incorporated in the consensus guidelines on $\mathrm{AD}$ and predementia AD-related conditions. So far, three different fluorine-18-labeled agents have been approved for clinical use in a variety of jurisdictions around the world. Despite these major advancements, Canada is not yet one of those jurisdictions. Publication of appropriate use criteria by the AA and the SNMMI has paved the way for other countries to adopt a standardized model reinforcing proper use of amyloid imaging in medical practice. This paper is derived from discussions of the STAC, a group of local, national and international dementia experts and imaging specialists who revisited the scenarios in which amyloid PET could be used appropriately in Canada. The final product is an updated set of guidelines to the 2012 CCCDTD4 effort, ${ }^{3}$ which also factors in the results of a survey of current practices in Canadian dementia centers. Furthermore, it is tailored to the Canadian reality and wishes to promote future development of amyloid imaging in our country. We hope this paper will generate momentum for the industry to submit a new drug proposal to Health Canada so that regulatory bodies approve the technique and discussions about provincial reimbursement can begin.

\section{Canadian Challenges}

This consensus effort allowed members to realize that only two Canadian provinces (Alberta and Quebec) have access to neurological FDG-PET examinations, a well-established molecular imaging technique in the field of dementia. Indeed, use of FDGPET has been supported by a wealth of literature (see Bohnen et $\mathrm{al}^{68}$ for a review). In the diagnosis of $\mathrm{AD}$, authors have showed that FDG-PET is superior to a baseline clinical evaluation and similar to an evaluation performed 4 years later. ${ }^{69}$ The addition of 


\section{Table 1: Recommendations for clinicians on behalf of the Canadian Consensus Conference on the Use of Amyloid Imaging}

1. Amyloid imaging represents a promising technique in the evaluation of dementia for which much has been learned over the past decade. It is not currently approved for clinical use in Canada. When it becomes available to Canadian clinicians, it must not be considered a routine test:

A. In accord with Appropriate Use Criteria for Amyloid PET, ${ }^{59,61}$ we recommend its use in patients with objectively confirmed cognitive impairments in whom there is diagnostic uncertainty* after a comprehensive clinical evaluation (mental status testing, laboratory tests, and structural brain imaging using MRI $\dagger$ ), and in whom knowledge of $\mathrm{A} \beta$ status is expected to provide a more precise diagnosis and alter management;

B. Clinicians who wish to obtain amyloid imaging should refer patients to dementia centers with an expertise in this technique, i.e. dementia expertsł with substantial clinical experience and practice in dementia care who work in conjunction with nuclear medicine specialists qualified in amyloid imaging;

C. We strongly recommend against the use of amyloid imaging in cognitively normal individuals or for the initial investigation of cognitive complaints.

2. Physicians should be cautious about interpreting the significance of amyloid test results, i.e. used in isolation this test cannot diagnose $\mathrm{AD}^{6,7} \mathrm{MCI},{ }^{8,33,34}$ or differentiate normal from abnormal aging. When faced with such situations, we recommend they consult with dementia centers with an expertise in this technique.

3. At present, there is no clinical indication for amyloid imaging in:

A. Attempting to differentiate AD from other a $\beta$-associated dementia (e.g. dementia with Lewy bodies, cerebral amyloid angiopathy);

B. Attempting to differentiate between AD clinical variants (e.g. classic amnestic AD vs. posterior cortical atrophy or logopenic variant of primary progressive aphasia);

C. Attempting to differentiate between the various clinical presentations associated with frontotemporal lobar degeneration spectrum of disorders (e.g. behavioral variant frontotemporal dementia vs progressive supranuclear palsy) to try to define the underlying pathology;

D. Staging the severity of a dementing syndrome.

4. Patients with $\mathrm{MCI}^{8,33,34}$ represent a highly heterogeneous cohort for which amyloid imaging may be appropriate under specific circumstances. As a general rule, amyloid PET could be considered in MCI patients for whom the dementia expert has determined that greater certainty about the underlying pathology would alter management (e.g. knowledge of amyloid burden in an individual $<65$ years old with confounding circumstances such as depression or other medical disorders, and for whom safety issues at work could have major consequences§). In such a case, determination of a positive amyloid status could lead to the diagnosis of MCI resulting from AD, as opposed to a nondegenerative condition, and have significant repercussions for future care and planning.

5. The actual process of undergoing an amyloid scan and the implications associated with disclosure of the results should be taken very seriously because this can be highly stressful for patients and families. To maximize safety and effectiveness of disclosing results, we recommend adopting parts of the sequence recently developed by Harkins et al ${ }^{65}$ in cognitively normal older adults participating in AD prevention studies. This format includes an educational session with clinical scenarios before the scan, assessment of mood and willingness to receive the results, and a formal face-to-face disclosure session in which results are discussed along with their diagnostic and prognostic implications.

$\mathrm{AD}=$ Alzheimer's disease; $\mathrm{MCI}=$ mild cognitive impairment; $\mathrm{MRI}=$ magnetic resonance imaging; $\mathrm{PET}=$ positron emission tomography.

*Subjects with diagnostic uncertainty after a comprehensive tertiary memory clinic evaluation are also named "complex/atypical cases" and often include $\mathrm{AD}$ variants, non- $\mathrm{AD}$ dementias (e.g. frontotemporal lobar degeneration), nonprogressing $\mathrm{AD}$, or patients with comorbid and nondegenerative conditions (e.g. depression, substance abuse, atypical bipolar disorder) (see Laforce \& Rabinovici ${ }^{66}$ for a detailed discussion).

$\dagger$ In accord with Canadian Consensus Conferences on the Diagnosis and Treatment of Dementia $4,{ }^{3}$ structural brain imaging using MRI means a "Head MRI - Dementia Protocol," which includes: (1) coronal T2/fluid-attenuated inversion recovery and (2) axial susceptibility-weighted imaging, diffusionweighted imaging, as well as three-dimensional T1 sequences. Structural brain imaging using MRI should always be performed before amyloid imaging as a positive scan in typical $\mathrm{AD}$ distribution in a patient with dementia can be secondary to cerebral amyloid angiopathy. ${ }^{67}$

$\ddagger$ Dementia expert: a physician with substantial clinical experience and practice in dementia care. Expertise in dementia is acquired through formal training and clinical experience in neurology, psychiatry, and geriatric medicine; however, not all dementia experts have expertise in amyloid imaging and/or work in conjunction with nuclear medicine specialists qualified in amyloid imaging, hence the recommendation to refer to a dementia center with expertise in this technique.

$\S$ Safety issues and the notion of major consequences: this is determined on a case-by-case basis with all significant clinical information. An example of this could be an emergency medical technician, paramedic, or a lead squad firefighter, etc.

FDG-PET to the investigation of atypical/unclear cases of dementia helps generate a more accurate diagnosis and initiate earlier treatment. ${ }^{47}$ Several studies have indicated that it is a cost- effective technique in the differential diagnosis of dementia ${ }^{70-73}$; yet, it appears that the vast majority of Canadian dementia centers do not take advantage of this technique.

\section{Table 2: Recommendations for research and translational development to clinical care on behalf of the Canadian Consensus Conference on Use of Amyloid Imaging}

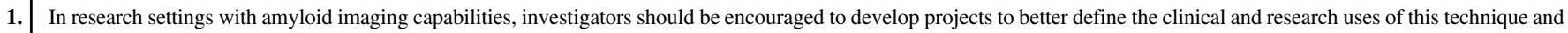
evaluate its readiness for translation to clinical care.

2. Trial designers are strongly encouraged to use this technique to (1) decrease the heterogeneity of their mild cognitive impairment population; (2) identify a cohort that is likely to respond to a drug with antiamyloid properties; and (3) study patients that are likely to convert to AD in a relatively short time frame.

3. Testing and longitudinal follow-up of asymptomatic individuals or patients with subjective cognitive impairments not meeting mild cognitive impairment criteria, or at-risk individuals (e.g. gene mutation carriers, family history of $\mathrm{AD}$, apolipoprotein $\mathrm{E} \varepsilon 4$ ) should be restricted to research.

4. Future research should explore (1) the natural evolution of amyloid burden and its role in the pathophysiology of AD and other dementias, (2) its use as a potential surrogate marker for anti-amyloid therapies, (3) pharmaco-economics issues of amyloid imaging, (4) positron emission tomography-pathology correlations, and (5) the links between amyloid imaging with cerebrospinal fluid AD biomarkers as well as downstream markers of neurodegeneration.
}

$\mathrm{AD}=$ Alzheimer's disease 
One could argue that amyloid imaging, when approved by regulatory bodies, will be in no different situation than FDG-PET because its use will also depend on provincial reimbursement policies. In 2013, our American colleagues faced a similar situation with regards to reimbursement for amyloid imaging. The Centers for Medicare and Medicaid Services (CMS) National Coverage Decision on amyloid PET imaging in dementia and neurodegenerative disease (CAG-00431N) ruled not to cover the scans because "the evidence is insufficient to conclude that the use of amyloid PET imaging is reasonable and necessary for the diagnosis or treatment of illness or injury or to improve the functioning of Medicare beneficiaries with dementia or neurodegenerative disease." CMS questioned the ability of amyloid PET to lead to improved health outcomes, such as avoidance of futile treatment or tests, improving or slowing the decline of quality of life, and survival. However, CMS did find sufficient evidence that the use of amyloid PET is promising: (1) to exclude AD in narrowly defined and clinically difficult diagnoses and (2) to enrich clinical trials seeking better treatments or prevention strategies for AD. The soon-to-be launched IDEAS study (discussed previously) was developed partly in response to this decision and it is hypothesized that it will demonstrate that knowledge of amyloid status leads to significant changes in patient management and improved medical outcomes, particularly in diagnostically uncertain cases of MCI and atypical dementia.

We believe time has come for Canadian dementia experts to take leadership in defining the role of molecular imaging in the differential diagnosis of dementia. Reimbursement issues surrounding the two PET techniques currently available should be discussed with appropriate provincial health authorities. Both techniques (FDG-PET and amyloid imaging) are now supported by a solid body of evidence, and politicians properly informed of their benefits should be open to fund programs where FDG-PET and amyloid PET are reimbursed in specific clinical situations along the guidelines detailed previously (e.g. differential diagnosis of AD vs FTLD).

\section{Amyloid Imaging versus CSF}

Amyloid imaging is not the sole biomarker which may signal underlying $\mathrm{AD}$ pathology. A decade or more before the appearance of dementia symptoms, and possibly before amyloid accumulation is detectable by PET, CSF changes can appear and correlate with brain atrophy in cognitively normal elderly. ${ }^{74-76}$ As suggested in the CCCDTD4 paper, ${ }^{3}$ authors have compared amyloid imaging with CSF AD biomarkers in the same study and found that CSF $\mathrm{A} \beta_{1-42}$ analyzed consecutively in routine clinical practice at an accredited laboratory can be used with high accuracy to determine whether a patient has normal or increased cortical A $\beta$ deposition and so can be valuable for the early diagnosis of AD. ${ }^{77}$ Other groups replicated these findings using cross-sectional and longitudinal designs. ${ }^{78}$ Interestingly, abnormal flutemetamol retention levels correlated with disease stage in patients with mild cognitive symptoms, but this was not the case for CSF $\mathrm{A} \beta_{1-42}{ }^{77}$

The utility of CSF in diagnosing unclear dementing syndromes such as those on the FTLD spectrum is less clear. ${ }^{79-81}$ Although tau fibrils and aggregates are pathological hallmarks of several FLTD subtypes, total CSF tau (t-tau) appears to be a general marker of neurodegeneration, whereas phosphorylated tau (i.e. p-tau-231 and p-tau-181) are useful in discriminating between AD and frontotemporal dementia. ${ }^{82}$ Increased ratio of tau/A $\beta_{1-42}$ can also distinguish AD from FTLD, ${ }^{83-85}$ and a low CSF $p / t$-tau ratio may distinguish FTLD-TDP from FTLD-tau. ${ }^{86}$ Another study suggests that total and p-tau in CSF were elevated in primary progressive aphasia relative to the behavioral variant frontotemporal dementia. ${ }^{87}$

At the moment, however, CSF variability across techniques and centers is such that it limits proper confident interpretation of the results. Because of the absence of appropriate laboratory infrastructure in Canada, or consensus as to where the samples should be sent for analysis, $A \beta_{1-42}$, t-tau, and p-181-tau have no clinical utility in Canada (not recommended for clinical practice), although they are part of research protocols in observational and therapeutic studies. Current international efforts to standardize CSF AD biomarkers, ${ }^{88}$ notably with more reliable enzyme-linked immunosorbent assay techniques, are currently under way.

\section{Action Plan}

An action plan was developed modeled on appropriate use criteria by Johnson and colleagues. ${ }^{59,61}$ These recommendations cover issues that could be disseminated to Canadian health care professionals and dementia organizations through knowledge translation activities: (1) who should be referred for an amyloid scan; (2) education (patients and families, health care professionals, dementia organizations); (3) amyloid PET scanning technique, interpretation (visual vs quantitative), translation into a clinical decision; and (4) proper disclosure of results.

\section{Who Should Be Referred for an Amyloid Scan?}

This decision should rely on the dementia expert guided by current Canadian guidelines. A dementia expert is a physician with substantial clinical experience and practice in dementia care. Expertise in dementia is acquired through formal training and clinical experience in neurology, psychiatry, and geriatric medicine; however, not all dementia experts have expertise in amyloid imaging and/or work in conjunction with a nuclear medicine specialist (NMS) qualified in amyloid imaging, hence the recommendation to refer to a dementia center with expertise in this technique, when appropriate.

\section{Education (Patients, Families, Health Care Professionals, Dementia Organizations)}

Dementia specialists in Canada are committed to disseminating information to the public and dementia organizations (e.g. information on amyloid imaging already exists on the Alzheimer Society's website at http://www.alzheimer.ca), to assisting colleagues in appropriate use of amyloid imaging, and in providing clarifications on how to incorporate amyloid PET in medical practice. Knowledge translation activities should be organized in all Canadian provinces to introduce the most recent guidelines on amyloid imaging.

Amyloid PET Scanning, Interpretation, and Translation Into a Clinical Decision

\section{Amyloid PET Scanning}

Imaging procedures should be performed by qualified nuclear medicine technologists and NMS with national certification in nuclear medicine and appropriate qualification in amyloid 
imaging. It should be performed in an imaging facility certified by Canadian accrediting agencies. Procedure guidelines for amyloid PET (SNMMI and European Association of Nuclear Medicine) should be followed.

\section{Interpretation}

The nuclear medicine team (i.e. the technologist and the NMS) performing the scan must be familiar with brain anatomy and must have adequate specific training in amyloid PET interpretation because amyloid PET imaging can be technically challenging and should be performed with strict attention to quality control. ${ }^{59,61}$ Web-based instruction programs have been developed and validated and should be completed successfully by all amyloid imaging teams before reading scans. Interpretation of amyloid PET imaging should be communicated to the referring physician by the NMS by way of a written report according to a standard diagnostic imaging practice as outlined in the SNMMI General Imaging Guideline. At this time, the final reading should indicate whether $A \beta$ was present (amyloid positive) or was not present (amyloid negative). The protocol for the qualitative read that determines positivity or negativity must be standardized $^{89}$ and must conform to a specific guideline provided by the manufacturer. Indeterminate results may arise as a result of technical or physiological factors and should be reported as such.

\section{Translation Into a Clinical Decision}

The NMS report should not equate amyloid positivity with AD dementia (amyloid positivity is not synonymous with $\mathrm{AD}$, and amyloid scans cannot replace a detailed clinical evaluation). Upon receiving the NMS report, the dementia expert should proceed to a comprehensive review of the clinical assessment (history, physical examination) and test results (laboratories, neurocognitive testing, MRI, FDG-PET) and incorporate amyloid results into a clinical decision process, always considering that amyloid imaging is an evolving modality and that image interpretation criteria, clinical significance of positive and/or negative scans, and technical imaging considerations are evolving. ${ }^{52}$

\section{Proper Disclosure of Results}

That moment can be highly stressful for patients and families. To maximize safety and effectiveness of disclosing results, we recommend consulting the sequence recently developed by Harkins et $\mathrm{al}^{65}$ in cognitively normal older adults participating in AD prevention studies. This process included: (1) an educational session, in which participants receive verbal and written information covering what is known and unknown about amyloid imaging, including possible results and their meaning; (2) screening for anxiety and depression to determine suitability to receive amyloid imaging information; (3) checking comprehension and recognizing distress; (4) conducting imaging on a separate day from consent, and disclosing results on a separate day from imaging; 5) proceeding to disclosure in person, with time for questions (at disclosure, physicians should assess mood and willingness to receive results); and 6) offering resources for support (brochures, follow-up call). The latter were developed for normal older adults participating in AD prevention studies. This may not be entirely possible in clinical practice, but can serve as general principles to guide proper disclosure.

\section{Future Challenges}

Amyloid imaging has been approved for widespread clinical use by leading health authorities in the United States and the United Kingdom. Despite this, several unknowns about its diagnostic utility remain and future studies should particularly focus on (1) its sensitivity and specificity as compared with pathology in practice-based settings (as opposed to the hospice studies), (2) technical and patient factors that could lead to false positives and false negatives, (3) the relative contribution of both diffuse and neuritic plaques' binding to the in vivo signal, (4) interpretation of the test as a dichotomous result versus assessing binding intensity and spatial distribution, (5) inter- and intra-rater reliability of visual and/or quantitative interpretations, (6) the optimal quantitative threshold for defining a positive scan for each of the available tracers, (7) whether the threshold for PiB positivity should be adjusted based on demographic factors such as age or genetic variables, and (8) cost effectiveness issues. Such issues are relevant for any diagnostic test, and should be addressed as research continues to target key variables associated with amyloid imaging. Recent longitudinal initiatives such as the IDEAS study should help answer several of these questions.

\section{Conclusions}

Amyloid imaging represents a major breakthrough in the evaluation of dementia that will doubtlessly translate into better clinical care and ultimately help guide the development of molecular-based therapies for these devastating illnesses. An impressive body of research has already been generated in the field, and studies of practical clinical applications are emerging with a specific indication in patients with objectively confirmed cognitive impairments where diagnostic uncertainty remains even after a comprehensive clinical evaluation in a tertiary memory clinic. This technique should always remain an adjunct imaging tool that is part of a comprehensive clinical evaluation in which a dementia expert determines that having a more accurate clinical diagnosis will alter management. Fundamentally, amyloid imaging detects a brain histological state, and is not a clinical diagnosis. Used in isolation, it cannot diagnose $\mathrm{AD}, \mathrm{MCI}$, or differentiate normal from abnormal aging. Clinical availability of new tracers in Canada would represent a major advancement for the many Canadians affected by an unclear dementing condition who suffer in silence while being exposed to unnecessarily prolonged delays before diagnosis, repeated and pointless visits and diagnostic tests with inferior sensitivity and specificity than amyloid imaging, and inappropriate treatments or lack thereof when indicated. In the end, we wish this effort to generate momentum for the industry to submit a new drug proposal to Health Canada so that regulatory bodies approve the technique and approval of provincial reimbursement can follow its proper course.

\section{ACKNOWLEDGEMENTS AND Funding}

Sincere thanks to the staff of the McGill Center for Studies in Aging who helped during the meeting in Montreal. We also thank the Molson Foundation and the Molson Lecture Series (http:// aging.mcgill.ca/talks.php) for their support and dissemination of knowledge. Financial support for the meeting was obtained from Le réseau des cliniques mémoire du Québec and the Ethical, Legal, Social Impact committee of the Canadian Consortium on Neurodegeneration in Aging funded by the Canadian Institutes of 
Health Research. Finally, sincere thanks to Société Alzheimer de Québec, Fondation du CHU de Québec, and Fonds sur la maladie d'Alzheimer de l'Université Laval.

\section{DiscLOSURES}

PR-N is an independent investigator and has a research Contract with Navidea Biopharmaceuticals. J-PS is a speaker and has received a speaker fee from Novartis. GR has been an invited speaker and received honoraria from GE Healthcare and Piramal Imaging; been a principal investigator/independent investigator and received research support from Avid Radiopharmaceuticals; and been a principal investigator and received research support from the Alzheimer's Association, NIH, Tau Consortium, and French Foundation. BD has been a consultant and received consulting fees from Eli Lilly. SG has been an advisor and received consulting fees from Lilly, TauRx, Ever Pharma, and Schwabe; and been a speaker and received speaker fees from Lundbeck and Schwabe. RL has nothing to disclose.

\section{REFERENCES}

1. Patterson CJ, Gauthier S, Bergman $\mathrm{H}$, et al. The recognition, assessment and management of dementing disorders: conclusions from the Canadian Consensus Conference on Dementia. CMAJ. 1999;160:S1-15.

2. Chertkow H. Diagnosis and treatment of dementia: introduction. Introducing a series based on the Third Canadian Consensus Conference on the Diagnosis and Treatment of Dementia. CMAJ. 2008; 178:316-21.

3. Gauthier S, Patterson C, Chertkow H, et al. 4th Canadian Consensus Conference on the Diagnosis and Treatment of Dementia. Can J Neurol Sci. 2012;39:S1-8.

4. Assessing dementia: the Canadian consensus. Organizing Committee, Canadian Consensus Conference on the Assessment of Dementia. Can Med Assoc J. 1991;144:851-3.

5. Dubois B, Feldman H, Jacova C, et al. Research criteria for the diagnosis of Alzheimer's disease: revisiting the NINCDSADRDA criteria. Lancet Neurol. 2007;6:734-46.

6. Dubois B, Feldman HH, Jacova C, et al. Revising the definition of Alzheimer's disease: a new lexicon. Lancet Neurol. 2010;9:1118-27.

7. McKhann G, Knopman D, Chertkow $\mathrm{H}$, et al. The diagnosis of dementia due to Alzheimer's disease: recommendations from the National Institute on Aging and the Alzheimer's Association workgroup. Alzheimers Dement. 2011;7:263-9.

8. Albert M, DeKosky S, Dickson D, et al. The diagnosis of mild cognitive impairment due to Alzheimer's disease: recommendations from the National Institute on Aging-Alzheimer's Association workgroups on diagnostic guidelines for Alzheimer's disease. Alzheimers Dement. 2011;7:270 9.

9. Sperling RA, Aisen PS, Beckett LA, et al. Toward defining the preclinical stages of Alzheimer's disease: recommendations from the National Institute on Aging-Alzheimer's Association workgroups on diagnostic guidelines for Alzheimer's disease. Alzheimers Dement. 2011;7:280-92.

10. Burhan A, Bartha R, Bocti C, et al. Role of emerging neuroimaging modalities in patients with cognitive impairment: a review from the Canadian Consensus Conference on the Diagnosis and Treatment of Dementia 2012. Alzheimers Res Ther. 2013;5:S4.

11. Soucy J-P, Bartha R, Bocti C, et al. Clinical applications of neuroimaging in patients with Alzheimer's disease: a review from the Fourth Canadian Consensus Conference on the Diagnosis and Treatment of Dementia 2012. Alzheimers Res Ther. 2013;5:S3.

12. Klunk WE, Engler H, Nordberg A, et al. Imaging brain amyloid in Alzheimer's disease with Pittsburgh Compound-B. Ann Neurol. 2004;55:306-19.

13. Jack CR, Jr., Wiste HJ, Weigand SD, et al. Amyloid-first and neurodegeneration-first profiles characterize incident amyloid PET positivity. Neurology. 2013;81:1732-40.
14. Sperling RA, Rentz DM, Johnson KA, et al. The A4 study: stopping AD before symptoms begin? Sci Transl Med. 2014;6:228fs13.

15. Farlow M, Arnold SE, van Dyck CH, et al. Safety and biomarker effects of solanezumab in patients with Alzheimer's disease. Alzheimers Dement. 2012;8:261-71.

16. Rinne JO, Brooks DJ, Rossor MN, et al. 11C-PiB PET assessment of change in fibrillar amyloid-beta load in patients with Alzheimer's disease treated with bapineuzumab: a phase 2, double-blind, placebo-controlled, ascending-dose study. Lancet Neurol. 2010;9:363-72.

17. Keller D. Finally, a big win for a monoclonal in Alzheimer's. AD/PD 2015: International Conference on Alzheimer's and Parkinson's Diseases 2015 [cited 2015, July 31]. Available from: http://www. medscape.com/viewarticle/841856.

18. Clark C, Pontecorvo M, Beach T, et al. Cerebral PET with florbetapir compared with neuropathology at autopsy for detection of neuritic amyloid-beta plaques: a prospective cohort study. Lancet Neurol. 2012;11:669-78.

19. Wolk DA, Grachev ID, Buckley C, et al. Association between in vivo fluorine 18-labeled flutemetamol amyloid positron emission tomography imaging and in vivo cerebral cortical histopathology. Arch Neurol. 2011;68:1398-403.

20. Rowe CC, Ackerman U, Browne W, et al. Imaging of amyloid beta in Alzheimer's disease with 18F-BAY94-9172, a novel PET tracer: proof of mechanism. Lancet Neurol. 2008;7:129-35.

21. Ossenkoppele R, Prins ND, Pijnenburg YA, et al. Impact of molecular imaging on the diagnostic process in a memory clinic. Alzheimers Dement. 2013;9:414-21.

22. Sanchez-Juan P, Ghosh PM, Hagen J, et al. Practical utility of amyloid and FDG-PET in an academic dementia center. Neurology. 2014;82:230-8.

23. Rabinovici G, Rosen H, Alkalay A, et al. Amyloid versus FDG PET in the differential diagnosis of AD and FTLD. Neurology. 2011;77:2034-42.

24. Wolk DA, Price JC, Madeira C, et al. Amyloid imaging in dementias with atypical presentation. Alzheimers Dement. 2012;8:389-98.

25. Bensaïdane M, Fortin M, Damasse G, Chenard M, Dionne C. Clinical utility of amyloid imaging in a complex case of corticobasal syndrome presenting with psychiatric symptoms. J Neurol Disord. 2014;2:2

26. Grundman M, Pontecorvo MJ, Salloway SP, et al. Potential impact of amyloid imaging on diagnosis and intended management in patients with progressive cognitive decline. Alzheimers Dis Assoc Disord. 2013;27:4-15.

27. Zannas AS, Doraiswamy PM, Shpanskaya KS, et al. Impact of 18F-florbetapir PET imaging of beta-amyloid neuritic plaque density on clinical decision-making. Neurocase. 2014;20:466-73.

28. Mitsis E, Bender H, Kostakoglu L, et al. A consecutive case series experience with $[18 \mathrm{~F}]$ florbetapir PET imaging in an urban dementia center: impact on quality of life, decision making, and disposition. Mol Neurodegener. 2014;9:10.

29. Bensaïdane MR, Beauregard JM, Poulin S, et al. (forthcoming). Clinical utility of amyloid PET Imaging in the differential diagnosis of atypical dementias and its impact on caregivers. J Alzheimers Dis. 2016.

30. Pike K, Savage G, Villemagne V, et al. Beta-amyloid imaging and memory in nondemented individuals: evidence for preclinical Alzheimer's disease. Brain. 2007;130:2837-44.

31. Rowe C, Ellis K, Rimajova M, et al. Amyloid imaging results from the Australian Imaging, Biomarkers and Lifestyle (AIBL) study of aging. Neurobiol Aging. 2010;31:1275-83.

32. Jack CR, Jr., Wiste HJ, Weigand SD, et al. Age-specific population frequencies of cerebral beta-amyloidosis and neurodegeneration among people with normal cognitive function aged 50-89 years: a cross-sectional study. Lancet Neurol. 2014;13:997-1005.

33. Petersen RC. Mild cognitive impairment as a diagnostic entity. J Intern Med. 2004;256:183-94.

34. Petersen RC. MIld cognitive impairment or questionable dementia? Arch Neurol. 2000;57:643-4.

35. Kantarci K, Lowe V, Przybelski SA, et al. APOE modifies the association between $A \beta$ load and cognition in cognitively normal older adults. Neurology. 2012;78:232-40. 
36. Mormino EC, Betensky RA, Hedden T, et al. Synergistic effect of beta-amyloid and neurodegeneration on cognitive decline in clinically normal individuals. JAMA Neurol. 2014;71:1379-85.

37. Dickerson BC, Bakkour A, Salat DH, et al. The cortical signature of Alzheimer's disease: regionally specific cortical thinning relates to symptom severity in very mild to mild AD dementia and is detectable in asymptomatic amyloid-positive individuals. Cereb Cortex. 2009;19:497-510.

38. Roe CM, Fagan AM, Grant EA, et al. Amyloid imaging and CSF biomarkers in predicting cognitive impairment up to 7.5 years later. Neurology. 2013;80:1784-91.

39. Klunk WE. Amyloid imaging as a biomarker for cerebral beta-amyloidosis and risk prediction for Alzheimer dementia. Neurobiol Aging. 2011;32:S20-36.

40. Ostrowitzki S, Deptula D, Thurfjell L, et al. Mechanism of amyloid removal in patients with Alzheimer disease treated with gantenerumab. Arch Neurol. 2012;69:198-207.

41. Pontecorvo M, Mintun M. PET amyloid imaging as a tool for early diagnosis and identifying patients at risk for progression to Alzheimer's disease. Alzheimers Res Ther. 2011;3:11.

42. Wolk DA, Price JC, Saxton JA, et al. Amyloid imaging in mild cognitive impairment subtypes. Ann Neurol. 2009;65:557-68.

43. Okello A, Koivunen J, Edison P, et al. Conversion of amyloid positive and negative MCI to AD over 3 years: an 11C-PIB PET study. Neurology. 2009;73:754-60.

44. Ellis KA, Lim YY, Harrington K, et al. Decline in cognitive function over 18 months in healthy older adults with high amyloid-beta. J Alzheimers Dis. 2013;34:861-71.

45. Rabinovici GD, Jagust WJ, Furst AJ, et al. Abeta amyloid and glucose metabolism in three variants of primary progressive aphasia. Ann Neurol. 2008;64:388-401.

46. de Souza LC, Corlier F, Habert MO, et al. Similar amyloid-beta burden in posterior cortical atrophy and Alzheimer's disease. Brain. 2011;134:2036-43.

47. Laforce R, Buteau J, Paquet N, Verret L, Houde M, Bouchard R. The value of PET in mild cognitive impairment, typical and atypical/ unclear dementias: a retrospective memory clinic study. Am J Alzheimers Dis Other Dement. 2010;25:324-32.

48. Ratnavalli E, Brayne C, Dawson K, Hodges JR. The prevalence of frontotemporal dementia. Neurology. 2002;58:1615-21.

49. Alladi S, Xuereb J, Bak T, et al. Focal cortical presentations of Alzheimer's disease. Brain. 2007;130:2636-45.

50. Villemagne VL, Ong K, Mulligan RS, et al. Amyloid imaging with (18)F-florbetaben in Alzheimer disease and other dementias. J Nucl Med. 2011:52:1210-7.

51. Beach TG, Monsell SE, Phillips LE, Kukull W. Accuracy of the clinical diagnosis of Alzheimer disease at National Institute on Aging Alzheimer Disease Centers, 2005-2010. J Neuropathol Exp Neurol. 2012;71:266-73.

52. Ossenkoppele R, Jansen WJ, Rabinovici GD, et al. Prevalence of amyloid pet positivity in dementia syndromes: a meta-analysis. JAMA. 2015;313:1939-50.

53. Cselényi Z, Jönhagen ME, Forsberg A, et al. Clinical validation of 18F-AZD4694, an amyloid- $\beta$-specific PET radioligand. J Nucl Med. 2012;53:415-24.

54. Juréus A, Swahn BM, Sandell J, et al. Characterization of AZD4694, a novel fluorinated $\mathrm{A} \beta$ plaque neuroimaging PET radioligand. J Neurochem. 2010;114:784-94.

55. Rowe CC, Pejoska S, Mulligan RS, et al. Head-to-head comparison of 11C-PiB and 18F-AZD4694 (NAV4694) for beta-amyloid imaging in aging and dementia. J Nucl Med. 2013;54:880-6.

56. Lee JH, Kim SH, Kim GH, et al. Identification of pure subcortical vascular dementia using 11C-Pittsburgh compound B. Neurology. 2011;77:18-25.

57. Johnson KA, Gregas M, Becker JA, et al. Imaging of amyloid burden and distribution in cerebral amyloid angiopathy. Ann Neurol. 2007;62:229-34.

58. Maetzler W, Liepelt I, Reimold M, et al. Cortical PIB binding in Lewy body disease is associated with Alzheimer-like characteristics. Neurobiol Dis. 2009;34:107-12.

59. Johnson K, Minoshima S, Bohnen N, et al. Appropriate use criteria for amyloid PET: a report of the Amyloid Imaging Task Force, the
Society of Nuclear Medicine and Molecular Imaging, and the Alzheimer's Association. Alzheimers Dement. 2013;9:1-15.

60. Jagust WJ. Amyloid imaging: liberal or conservative? Let the data decide. Arch Neurol. 2011;68:1377-8.

61. Johnson KA, Minoshima S, Bohnen NI, et al. Update on appropriate use criteria for amyloid PET imaging: dementia experts, mild cognitive impairment, and education. J Nucl Med. 2013;54: $1011-3$.

62. Curtis C, Gamez JE, Singh U, et al. Phase 3 trial of flutemetamol labeled with radioactive fluorine 18 imaging and neuritic plaque density. JAMA Neurol. 2015;72:287-94.

63. Sabri O, Sabbagh MN, Seibyl J, et al. Florbetaben PET imaging to detect amyloid beta plaques in Alzheimer's disease: phase 3 study. Alzheimers Dement. 2015;11:964-74.

64. Guyatt GH, Oxman AD, Vist GE, et al. GRADE: an emerging consensus on rating quality of evidence and strength of recommendations. BMJ. 2008;336:924-6.

65. Harkins K, Sankar P, Sperling R, et al. Development of a process to disclose amyloid imaging results to cognitively normal older adult research participants. Alzheimers Res Ther. 2015;7:26.

66. Laforce R, Rabinovici G. Amyloid imaging in the differential diagnosis of dementia: review and potential clinical applications. Alzheimers Res Ther. 2011;3:31.

67. Ducharme S, Guiot M-C, Nikelski J, Chertkow H. Does a positive Pittsburgh Compound B scan in a patient with dementia equal Alzheimer disease? JAMA Neurol. 2013;70:912-4.

68. Bohnen N, Djang D, Herholz K, Anzai Y, Minoshima S. Effectiveness and safety of 18F-FDG PET in the evaluation of dementia: a review of the recent literature. J Nucl Med. 2012;53:59-71.

69. Jagust W, Reed B, Mungas D, Ellis W, Decarli C. What does fluorodeoxyglucose PET imaging add to a clinical diagnosis of dementia? Neurology. 2007;69:871-7.

70. Mosconi L. Brain glucose metabolism in the early and specific diagnosis of Alzheimer's disease. FDG-PET studies in MCI and AD. Eur J Nucl Med Mol Imaging. 2005;32:486-510.

71. Mosconi L, Tsui WH, Herholz K, et al. Multicenter standardized 18F-FDG PET diagnosis of mild cognitive impairment, Alzheimer's disease, and other dementias. J Nucl Med. 2008;49:390-8.

72. Silverman D, Small G, Chang C, et al. Positron emission tomography in evaluation of dementia - regional brain metabolism and longterm outcome. JAMA. 2001;286:2120-7.

73. Silverman DH, Gambhir SS, Huang HW, et al. Evaluating early dementia with and without assessment of regional cerebral metabolism by PET: a comparison of predicted costs and benefits. J Nucl Med. 2002;43:253-66.

74. Jack C, Wiste H, Vemuri P, et al. Brain beta-amyloid measure and magnetic resonance imaging atrophy both predict time-toprogression from mild cognitive impairment to Alzheimer's disease. Brain. 2010;133:3336-48.

75. Bateman RJ, Xiong C, Benzinger TL, et al. Clinical and biomarker changes in dominantly inherited Alzheimer's disease. N Engl J Med. 2012;367:795-804.

76. Fagan AM, Head D, Shah AR, et al. Decreased CSF A $\beta 42$ correlates with brain atrophy in cognitively normal elderly. Ann Neurol. 2009;65:176-83.

77. Palmqvist S, Zetterberg H, Blennow K, et al. Accuracy of brain amyloid detection in clinical practice using cerebrospinal fluid beta-amyloid 42: a cross-validation study against amyloid positron emission tomography. JAMA Neurol. 2014;71:1282-9.

78. Landau SM, Lu M, Joshi AD, et al. Comparing positron emission tomography imaging and cerebrospinal fluid measurements of beta-amyloid. Ann Neurol. 2013;74:826-36.

79. Hampel H, Bürger K, Teipel SJ, Bokde AL, Zetterberg H, Blennow $\mathrm{K}$. Core candidate neurochemical and imaging biomarkers of Alzheimer's disease. Alzheimers Dement. 2008;4:38-48.

80. Mulder C, Verwey NA, van der Flier WM, et al. Amyloid-beta(1-42), total tau, and phosphorylated tau as cerebrospinal fluid biomarkers for the diagnosis of Alzheimer disease. Clin Chem. 2010;56:248-53.

81. Rosa-Neto P, Hsiung G-Y, Masellis M, on behalf of the CCDTD4 participants. Fluid biomarkers for diagnosing dementia: rationale and the Canadian Consensus on Diagnosis and Treatment of 
Dementia recommendations for Canadian physicians. Alzheimers Res Ther. 2013;5:S8.

82. Hampel H, Teipel SJ. Total and phosphorylated tau proteins: evaluation as core biomarker candidates in frontotemporal dementia. Dement Geriatr Cogn Disord. 2004;17:350-4.

83. Bian H, Van Swieten JC, Leight S, et al. CSF biomarkers in frontotemporal lobar degeneration with known pathology. Neurology. 2008;70:1827-35.

84. Borroni B, Malinverno M, Gardoni F, et al. Tau forms in CSF as a reliable biomarker for progressive supranuclear palsy. Neurology. 2008;71:1796-803.

85. Kapaki E, Paraskevas GP, Papageorgiou SG, et al. Diagnostic value of CSF biomarker profile in frontotemporal lobar degeneration. Alzheimer Dis Assoc Disord. 2008;22:47-53.

86. Hu WT, Watts K, Grossman M, et al. Reduced CSF p-tau181 to tau ratio is a biomarker for FTLD-TDP. Neurology. 2013;81: 1945-52.

87. Bibl M, Mollenhauer B, Lewczuk P, et al. Cerebrospinal fluid tau, p-tau 181 and amyloid-beta38/40/42 in frontotemporal dementias and primary progressive aphasias. Dement Geriatr Cogn Disord. 2011;31:37-44.

88. Global Biomarker Standardization Consortium. Biomarker Standardization Projects. 2015 [cited 2015, Aug 29]; Available from: http://www.alz.org/research/funding/global_biomarker_projects. asp.

89. Landau SM, Mintun MA, Joshi AD, et al. Amyloid deposition, hypometabolism, and longitudinal cognitive decline. Ann Neurol. 2012;72:578-86.

\section{APPENDix A: Members}

\section{A. Specialized Task Force on Amyloid imaging in Canada (STAC)}

\section{Organizing Committee Members}

Serge Gauthier, MD (chair)

Robert Laforce Jr, MD, PhD

Pedro Rosa-Neto, MD (co-chair)

Jean-Paul Soucy, MD

\section{Invited International Leaders \\ Bruno Dubois, MD \\ Gil D. Rabinovici, MD}

\section{B. Consensus meeting participants}

Clinical and Academic

Howard Chertkow, MD

Guy Lacombe, MD

Alain Robillard, MD

Sylvia Villeneuve, $\mathrm{PhD}$

C. Member from Canadian Regulatory Bodies (Health Canada)

Agnes Klein, MD

D. Leading representatives from the molecular imaging and imaging software industries

Susan De Santi, PhD

Benoît Galarneau

Rick Hiatt

René Rebeaud

Dwight Tapp

\section{APPENDiX B: Relationships With IndUSTRY AND MANAGEMENT OF CONFLICTS OF INTEREST}

The group rigorously attempted to avoid any actual, perceived, or potential conflicts of interest that might have arisen as a result of an outside relationship or personal interest of the writing committee members. We reviewed our own industry relationship policies to ensure that the ensuing process adhered to current standards. Members were required to provide disclosure statements of all relationships that might be perceived as real or potential conflicts of interest. These statements were reviewed by the chair and senior author of this paper. 